\title{
Relating Linear and Volumetric Variables through Body Scanning to Improve Human Interfaces in Space
}

\author{
Sarah Margerum ${ }^{\mathrm{a}, \mathrm{d}}$, Mike Ferrer ${ }^{\mathrm{b}, \mathrm{d}}$, Karen Young $^{\mathrm{a}, \mathrm{d}}$, and Sudhakar Rajulu ${ }^{* c, d}$, Ph.D. \\ aLockheed Martin, Houston (TX), USA; \\ ${ }^{\mathrm{b}} \mathrm{MEI}$ Technologies Inc., Houston (TX), USA; \\ ${ }^{c}$ National Aeronautics and Space Administration, Johnson Space Center, Houston (TX), USA;

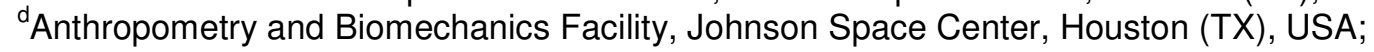

\begin{abstract}
Designing spacesuits and vehicles for the diverse human population presents unique challenges for the methods of traditional anthropometry. Existing spacesuits are bulky, allowing the operator to shift position within the suit and inhibiting the ability to identify body landmarks. Limited suit sizing options cause differences in fit and performance between similarly sized individuals. Space vehicles are restrictive in volume with respect to both the fit of astronauts and the ability to collect data on fit and mobility. NASA's Anthropometry and Biomechanics Facility (ABF) has shifted from using traditional linear anthropometry to exploring the capabilities of 3D scanning to provide volumetric anthropometric solutions for design. The key goals are to improve the human-system performance and develop new processes to aid in the design and evaluation of space systems. Four case studies are presented that illustrate the shift from purely linear analyses to an augmented volumetric tool set for predicting and analyzing the human within the spacesuit and vehicle.
\end{abstract}

The first case study involves the calculation of maximal head volume of the target population to estimate total free volume in the helmet for proper air exchange. Traditional linear measurements resulted in an inaccurate representation of the head shape, yet limited data exist for the determination of a large head volume. Steps were taken to identify and classify a maximal head volume. The resulting comparisons to the estimate are presented in this paper. This study illustrates the gap between linear components of anthropometry and the need for overall volume metrics to provide solutions.

A second case study examines the overlay of the spacesuit scans and components onto scanned individuals to quantify fit and clearance; to aid in sizing the suit to the individual. Restrictions in spacesuit size availability present unique challenges to optimally fit the individual within a limited sizing range while maintaining performance. Quantification of the clearance and fit for similarly sized individuals is critical in providing a greater understanding of the human body's function within the suit.

The third case study explores the development of a conformal seat pan using scanning techniques and details the challenges of volumetric analyses that were overcome to develop a universal seat pan that can be used across the entire user population.

The final case study explores expanding volumetric capabilities through generation of boundary manikins. Boundary manikins were developed as representative individuals from the population of interest that represent the extremes of the population spectrum. The ABF developed a technique to take 3D scans of individuals and manipulate the scans to reflect the boundary manikins' anthropometry. In essence, this process generates a representative 3D scan of an individual from anthropometry, using another individual's scanned image. The results from this process can be used in design process modeling and initial suit sizing work as a three-dimensional, realistic example of individuals from the population, maintaining the variability between and correlation with the dimensions of interest.

Keywords: volume, anthropometry, scanning, morphing, space

\section{Introduction}

The improvement of human-system interactions, the evaluation of accommodation parameters, and the development of new processes to aid in the design and evaluation of space systems are primary goals of the Anthropometry and Biomechanics Facility (ABF) at NASA Johnson Space Center. The

* sudhakar.rajulu-1@nasa.gov; 281-483-3725; http://hefd.jsc.nasa.gov/abf.htm 
complexities of traditional human-factors analyses are compounded due to the effects of space hardware, environment, and operations. For example, spacesuits restrict movement and comfort based on the size and fit of the individual. Total space vehicle mass is restricted and volume is at a premium to provide storage, crew facilities, and workspace for the crew for the duration of their mission. The need to place the highly variable human body within these restrictive physical environments to ensure that the entire anticipated population can live, work, and interact has led to a shift from evaluations focused on linear-based metrics to the development of volumetric tools to evaluate and analyze the human within the space environment.

This exploration into the capabilities of 3D scanning to provide volumetric anthropometric solutions for design arose when the linear measurements were found to be insufficient to quantitatively classify the subject within the population due to the multivariate nature of the problem itself. As exploration progressed, it became apparent that volumetric capabilities were a powerful tool to aid in the design of human-system interfaces. Four case studies are presented that illustrate the shift from purely linear analyses to an augmented volumetric tool set for predicting and analyzing the human within the spacesuit and vehicle.

\section{Case Study \#1: Maximal Head Volume}

The first case study involves the maximum and mean volume of the head-neck complex to estimate total free volume within the spacesuit helmet for the current astronaut population. The available traditional linear measurements were not sufficient to describe the shape properly. This study demonstrates the need for overall volume metrics to provide solutions and how the linear dimensions were used to assist in the identification and classification of the representative head volumes.

\subsection{Background}

The design of spacesuit helmet is to protect the crew from injury and from the external environment, while minimizing the impact on crew performance, field of view, and storage mass and volume. While the spacesuit is pressurized, the helmet also has a minimal free volume required for proper air exchange for the crewmember. Free volume is the total space remaining within a system that is not displaced by an astronaut. While regression equations can be used for derivation of this value [1], the interest was in the actual maximal head volume for crewmembers within the astronaut corps.

\subsection{Methods}

An initial attempt was made to estimate the total free volume using traditional linear measurements. Head length and head breadth, the only available head-specific anthropometry measurements from the population of interest, were used to approximate the volume using an oblate ellipsoid shape using these two measurements as the semi-principal axes, and using head length as the third axis to generate the ellipsoid. Using this equation of an ellipsoid, the maximal volume was initially calculated using a combination of the maximal head length and head breadth from the astronaut database. Unfortunately, the ellipsoid shape is not closely representative of the head (Figure 1) and it is highly unlikely that it represents the largest anticipated head volume. In addition to the inaccuracy of relating an ellipsoid to the shape of the head, it is inappropriate that the maximal values of the two measurements are used, due to the only moderate correlation $\left(r^{2}=0.4\right)$. Finally, the neck volume is completely ignored using this methodology. To ensure an accurate, reasonable maximal head volume, the linear dimensions of population were examined and the associated maximal volumes were determined from the representative 3D scans of crewmembers (VITUS/Smart ${ }^{\mathrm{TM}}$, Vitronic $\mathrm{GmbH}$, Wiesbaden, Germany).
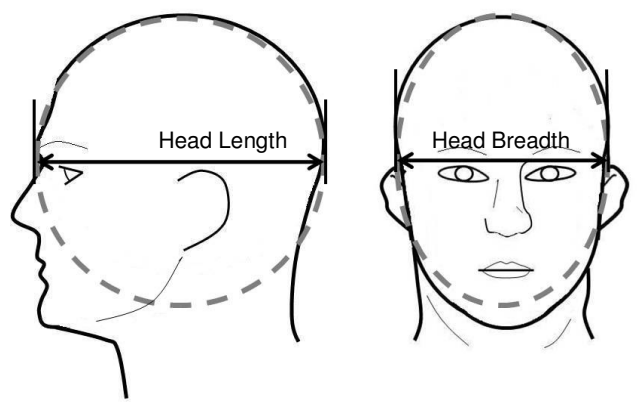

Fig. 1. The ellipsoid created by head length and head breadth. 
This move from examination of linear aspects of the body to considering the multivariate aspects using the volumetric data was limited in scope. The new methodology focused on associating the maximal anthropometric values with the actual volumes from 3D head scans. The ABF maintains anthropometric databases of key values used to assist in sizing the astronauts in the spacesuit and vehicle [2]. The ABF is also in the process of collecting 3D body scans of all current crewmembers. The head length and head breadth data for all current crewmembers was summarized and the mean and maximum head dimensions were retrieved from the astronaut databases. It was postulated that the maximal head volume would belong to an individual with some combination of the head breadth and head length values near the higher end of the population spectrum. The subjects that had the maximal or near-maximal head dimensions were then compared to the available 3D body scan repository in the $A B F$. If the crewmember had not yet been scanned by the ABF, an existing scan from the Civilian American and European Surface Anthropometry Resource (CAESAR) [3,4] of that astronaut was used for volume calculations.

\subsection{Results}

The maximum head length for all subjects in the database was $24.9 \mathrm{~cm}(9.8 \mathrm{in})$ for the males and 20.3 $\mathrm{cm}(8.0 \mathrm{in})$ for females (Table 1). However, 3D scans were not available for the male subject or for a corresponding CAESAR scan, so the next largest head length measurement that was available was $23.1 \mathrm{~cm}(9.1 \mathrm{in})$ for the males. The same method was repeated to determine the subjects with the mean head length, maximum head breadth, and the mean head breadth. The mean values were examined in conjunction to the maximum to investigate the overall difference in scope between maximum and the average volumes. The analysis of the database yielded three males representing maximum head length, max head breadth, mean head breadth and mean head length, and two females representing the maximum head dimensions and the mean head dimensions for analysis.

Table 1. Maximum and mean head dimensions of the available crewmember anthropometry.

\begin{tabular}{|l|c|c|c|c|}
\hline & \multicolumn{2}{|c|}{ Head Length [cm (in)] } & \multicolumn{2}{c|}{ Head Breadth [cm (in)] } \\
\hline & Mean & Max & Mean & Max \\
\hline Male & $20.1(7.9)$ & $24.9(9.8)$ & $16.0(6.3)$ & $18.3(7.2)$ \\
\hline Female & $19.1(7.5)$ & $20.3(8.0)$ & $14.7(5.8)$ & $16.5(6.5)$ \\
\hline
\end{tabular}

The 3D scans for these five subjects were processed using Polyworks (InnovMetric Software, Quebec, Canada). To calculate the volume of the scan images, the head and neck region was isolated using a plane at the neck, then removing the lower body. There were several holes (missing data points) in the scan images that needed to be filled in manually to create a watertight object before the volume could be computed. The holes in the scans are primarily due to the limitations of the scanner from the horizontal laser beam, causing holes around the ears, top of the head and bottom of the chin. Once all the holes were filled in and the image was made watertight (Figure 2), the Polyworks software was used to calculate the volume of the combined head and neck. The resulting volumes are displayed in Table 2. The largest volume was 5.5 liters $\left(338.5 \mathrm{in}^{3}\right)$. The difference between the maximum and mean volume values for males was 0.5 liters $\left(28.6 \mathrm{in}^{3}\right)$.

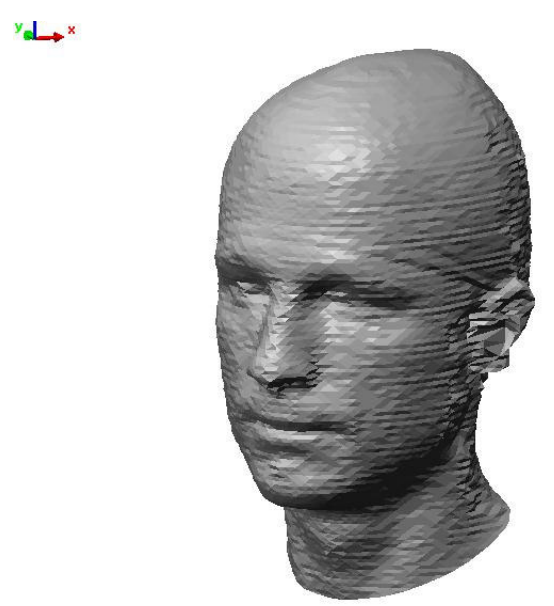

Fig. 2. A representative example of a scanned head image (source: CAESAR [3,4]). 
Table 2. Head volume results from Polyworks scan.

\begin{tabular}{|c|c|l|c|c|c|}
\hline $\begin{array}{c}\text { Subject } \\
\text { Number }\end{array}$ & Gender & Representative of: & $\begin{array}{c}\text { Head Length } \\
{[\mathrm{cm}(\mathrm{in})]}\end{array}$ & $\begin{array}{c}\text { Head Breadth } \\
{[\mathrm{cm}(\mathrm{in})]}\end{array}$ & $\begin{array}{c}\text { Volume } \\
\left.\left[\text { liters (in }{ }^{3}\right)\right]\end{array}$ \\
\hline 1 & $\mathrm{M}$ & Max Head Breadth & $19.0(7.5)$ & $18.2(7.2)$ & $5.3(325.7)$ \\
\hline 2 & $\mathrm{M}$ & Max Head Length & $23.0(9.1)$ & $15.3(6.0)$ & $5.5(338.5)$ \\
\hline 3 & $\mathrm{M}$ & $\begin{array}{l}\text { Mean Head Length and } \\
\text { Mean Head Breadth }\end{array}$ & $20.0(7.9)$ & $15.9(6.3)$ & $5.1(309.9)$ \\
\hline 4 & $\mathrm{~F}$ & $\begin{array}{l}\text { Max Head Length and } \\
\text { Head Breadth }\end{array}$ & $20.2(8.0)$ & $15.5(6.1)$ & $4.8(293.7)$ \\
\hline 5 & $\mathrm{~F}$ & $\begin{array}{l}\text { Mean Head Length and } \\
\text { Mean Head Breadth }\end{array}$ & $19.0(7.5)$ & $14.7(5.8)$ & $4.4(267.1)$ \\
\hline
\end{tabular}

\subsection{Summary}

The results yielded a maximal current crew head volume of 5.5 liters $\left(338.5 \mathrm{in}^{3}\right)$. In a comparison with the ellipsoidal head volume calculation using the maximum head breadth and head length values as the semi-principal axes of the ellipsoid, the current crew maximal volume is 0.4 liters $\left(23.6 \mathrm{in}^{3}\right)$ smaller than estimated (Table 3). This difference was unexpected as it was anticipated that the calculated volume from the 3D scan would be larger than the ellipsoidal calculations. However, this is explained by the use of both maximum head breadth and head length values as inputs into the ellipsoid equation. If the head measurements from the maximal subject (Subject 2) are entered into the equation instead, the volume drops down to 4.2 liters $\left(258.7 \mathrm{in}^{3}\right)$. The volume of the ellipse should logically fall within that of the 3D scan head volume because the ellipse is contained within the head shape and excludes the neck. As previously discussed, using both the maximum head breadth and head length values is inappropriate due to the low correlation between the two measurements $\left(r^{2}=0.4\right)$.

To compare the results within the context of the anticipated crew population, the total head and neck volume was also calculated using the regression equations available in McConville (1980) [1]. The male stature and weight values from a modified age-truncated Anthropometric Survey of U.S. Army Personnel (ANSUR) database [5,6] were used as inputs to the regression equations for the head and neck volumes. The head and neck volumes were then summed for each male in the database and the mean and standard deviation were determined from the resulting volume values. The mean and standard deviation were used to calculate a predicted total head and neck volume of a $99^{\text {th }}$ percentile male and then compared to the current crew total head and neck volume. The current crew maximal head-neck volume is 0.5 liters $\left(28.5 \mathrm{in}^{3}\right)$ smaller than the $99^{\text {th }}$ percentile volume value of the head-neck using the regression equations (Table 3). This difference is consistent and anticipated, as the 3D scans used from the crew database do not represent the $99^{\text {th }}$ percentile male, only the maximal current crew data.

Table 3. Comparison of results against alternative calculations.

\begin{tabular}{|c|c|c|c|}
\hline & $\begin{array}{c}\text { Prediction based on } \\
\text { Maximum Ellipsoid } \\
{\left[\text { liters }\left(\text { in }^{3}\right)\right]}\end{array}$ & $\begin{array}{c}\text { Predicted 99th percentile } \\
\text { based on Regression Eqn. } \\
\left.\text { [liters }\left(\operatorname{in}^{3}\right)\right]\end{array}$ & $\begin{array}{c}\text { Actual Current Crew } \\
\text { Maximal 3D Scan Data } \\
\left.\text { [liters }\left(\mathrm{in}^{3}\right)\right]\end{array}$ \\
\hline Volume & 5.9 (362.1) & $6.0(367.0)$ & 5.5 (338.5) \\
\hline
\end{tabular}

This study illustrates the gap between linear components of anthropometry and ability to relate linear to volumetric variables. While linear regressions available through literature could be employed to derive volumetric information, the availability of $3 \mathrm{D}$ scan information provides a quick, easy, and accurate means for calculation of volume.

\section{Case Study \#2: Volumetric Overlay of Suit Components}

When examining the complex shape of the human body, linear measurements do not provide the entire picture. Placement and comparison of subjects within the context of the user population using just a single linear dimension is fairly simple; however, as more and more dimensions are added into the problem scope, it becomes difficult to define and differentiate the subjects within the population as a whole. There are techniques to parse and group a user population multivariately using such methods as Principal Component Analysis (PCA) [7] and cluster analysis [8] techniques, yet those are used for larger population-based analyses, not examination of a small subset of subjects. A 3D scan allows for 
that immediate relation of linear to multivariate dimensions of the human body using the volumetric aspects of the body scans. This section of the paper examines several ways the ABF has used whole-body scanner technology to evaluate suit fit.

\subsection{Evaluation of fit across individuals}

As the space program develops new suits, there is a need to verify and validate the suit fit, mobility, and performance using prototypes. In the early stages of design, prior to a suit sizing scheme, selection of the suit components is often geared toward the anthropometry of available suit test subjects. In this case, three subjects were selected on the basis of similar anthropometric parameters, a small selection of which is shown in Table 4. These subjects were selected for the intended purpose of using them as primary test subjects to operate the suit to perform prototype suit evaluations.

Table 4. Torso-based anthropometry of the test subjects.

\begin{tabular}{|c|c|c|c|c|c|c|}
\hline Subject & $\begin{array}{c}\text { Stature } \\
{[\mathrm{cm}(\text { in)] }}\end{array}$ & $\begin{array}{c}\text { Vertical Trunk } \\
\text { Diameter } \\
{[\mathrm{cm}(\text { in)] }}\end{array}$ & $\begin{array}{c}\text { Chest } \\
\text { Breadth } \\
\text { [cm (in)] }\end{array}$ & $\begin{array}{c}\text { Expanded } \\
\text { Chest Depth } \\
{[\mathrm{cm}(\text { in)] }}\end{array}$ & $\begin{array}{c}\text { Hip Breadth } \\
{[\mathrm{cm} \text { (in)] }}\end{array}$ & $\begin{array}{c}\text { Waist Depth } \\
{[\mathrm{cm} \text { (in)] }}\end{array}$ \\
\hline 1 & $164.6(64.8)$ & $63.8(25.1)$ & $31.5(12.4)$ & $26.9(10.6)$ & $38.9(15.3)$ & $21.6(8.5)$ \\
\hline 2 & $174.0(68.5)$ & $69.3(27.3)$ & $33.3(13.1)$ & $28.4(11.2)$ & $33.3(13.1)$ & $23.6(9.3)$ \\
\hline 3 & $167.4(65.9)$ & $62.7(24.7)$ & $29.0(11.4)$ & $24.9(9.8)$ & $34.5(13.6)$ & $21.6(8.5)$ \\
\hline Range & $9.1(3.6)$ & $6.6(2.6)$ & $4.3(1.7)$ & $3.6(1.4)$ & $5.6(2.2)$ & $2.0(0.8)$ \\
\hline
\end{tabular}

Examination of the values in Table 4 indicated that the subjects were extremely similar, with just 2.0 $\mathrm{cm}(0.8 \mathrm{in})$ to $6.6 \mathrm{~cm}$ (2.6 in) of difference for torso specific measurements. However, because the suit is such a restrictive fit, how large of a range is too large? How do these measurements combine to create the overall body shape? The ABF overlaid the torsos of the three test subjects on top of one another (Figure 3) to examine this combination of measurements and how they would impact the overall fit. In comparing all the subjects in this manner, it was observed that there was a large gap in the shoulder area (Figure 3 far left) and gaps at the hips and buttock area (Figure 3 middle) where there is a single subject that exceeds the other two subjects. In addition, the smallest subject would have large amounts of clearance at every point (Figure 3 middle and far right), meaning they would be 'swimming' inside the suit. The most problematic area to designers was the mid torso and chest. Suit components, such as drinking water bags, are placed in this area since the waist naturally tapers and provides a pocket to place those components. If a suit was made that fit to the external shape of the combined torsos, this pocket would need to be located right at the waist area, due to the differences observed in the chest and varying locations of the bustpoint of the multiple subjects. This location would have hindered waist mobility and/or the placement of waist disconnects for ingress and egress of the suit. Ultimately based on these results, the prototype design was delayed to develop a better sizing scheme and determine the acceptable variation ranges in anthropometry as it correlates to performance and mobility.
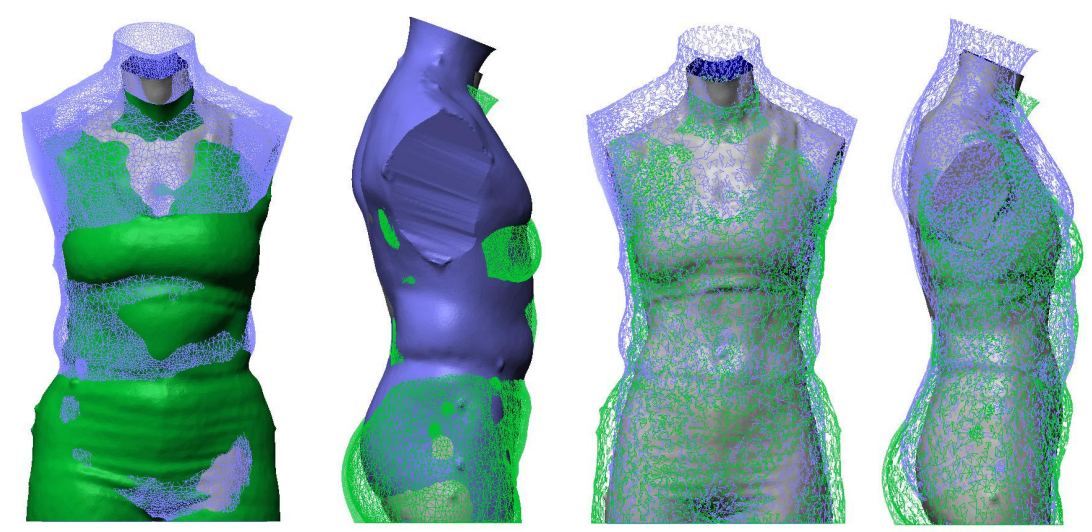

Fig. 3. Overlaid torsos of 3 test subjects, Subject 1 (green), Subject 2 (purple), and Subject 3 (gray).

\subsection{Evaluation of suit components}

The development of new suits also allows for computer-aided design (CAD) modeling of suit components. Previously, many suit designs predated the use of CAD modeling. As the space program develops new suits, the capabilities of CAD design can be used to evaluate fit and some performance 
issues in addition to providing feedback before the prototype is built, saving time and resources. The benefits of fitting the suit using the assistance of a CAD model is that it provides the entire picture of the interplay between the suit components and the variables that are important for sizing.

Restrictions in spacesuit size availability present unique challenges to optimally fit the individual within a limited sizing range while maintaining performance. For example, the hard upper torso (HUT) of a suit (Figure 4) has four bearings/disconnects that are the same dimensions across suit sizes, to maintain the modular connection of adding suit components for optimal sizing. While the shoulder rings do not change in diameter, their placement with respect to the mid-line of the HUT does change across sizes. For smaller HUT's (Figure 4 left) the shoulders are brought in more to accommodate a smaller person's shoulder breadth, anterior interscye breadth, and chest breadth. The placement of these shoulder bearings ideally would fall at the mid-shoulder landmark of the body. Finally, restrictions imposed on measurements such as the chest breadth and depth also impact the fit, from just a sheer accommodation standpoint (Figure 4 left, middle).

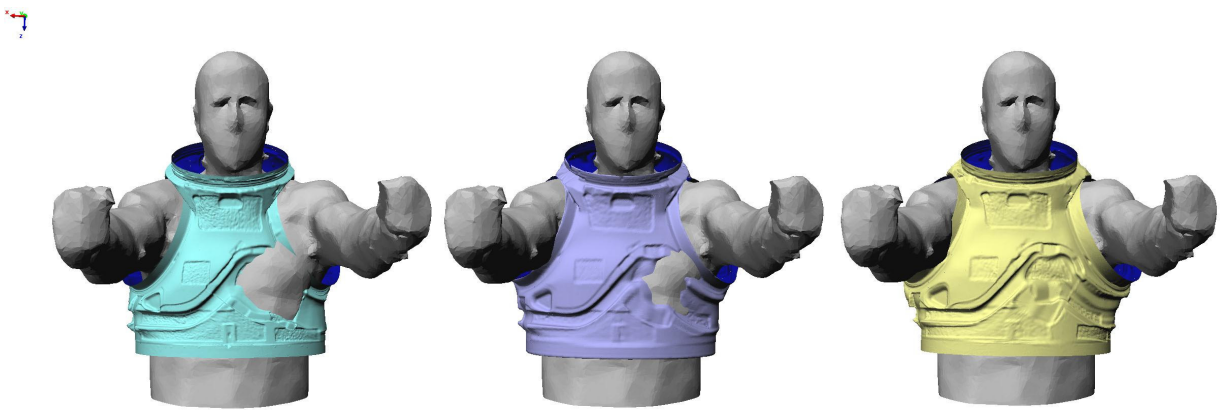

Fig. 4. Hard Upper Torsos overlaid onto a scanned subject: Medium (left), Large (middle), and X-Large (right).

The complex multivariate nature of the body causes trade-offs to occur with the overall fit of the suit based on the linear dimensions. A single measurement can cause an individual to jump to the next suit size, with the unintended consequences of a performance detriment. For example, the placement of the shoulder bearings for a person with a large mid-shoulder breadth may necessitate that an individual jump to a larger size according to the sizing schema. However, if that same individual has a small chest breadth and bideltoid breadth, the mobility at the shoulder is reduced because of the bearing impingement near the armpit. The ability to place a scan of the subject in CAD and add suit components can allow a suit designer to evaluate the impact and tradeoffs within the context of the overall linear anthropometry.

\subsection{Conclusion}

Anthropometric measurements provide the foundation for sizing a suit to an individual, yet there is always the potential to improve the comfort and fit of the suit using advancements in available technologies. The utility of the multivariate nature of the 3D body scanner can be used to generate immediate relationships to linear dimensions of the human body using the volumetric aspects of the body scans.

\section{Case Study \#3: Conformal Seat Pan}

Safety is of utmost importance in all aspects of the human space program. With this in mind, capturing the curvature of the lower-body of all potential users enables design of a universal seat pan with appropriate seat pads to accommodate the entire user population. The contour of the lower body of individuals cannot be described with conventional anthropometry; however, the linear measurements can be related to surface scans for evaluation of the seat-pan contour. In this study the lower body of individuals was captured, compared to the population, and then incorporated into the design of the universal seat-pan contour to properly integrate with the body shapes of future crewmembers that are flying in the space vehicle.

\subsection{Background}

A vital component of the design of a cockpit is the design of the interface between the human and the system. The space vehicle seat must be comfortable during long hours of operation and provide safety 
and support during launch and re-entry while accommodating the entire user population. Minimizing space and weight is crucial to spaceflight hardware, so cushioning and padding must be kept to a minimum. Accommodation of the seat interface to the user allows for the safety and comfort of the individual, which then reciprocates in successful mission task performance. Capturing and exporting surface-scans of individuals in a shirt-sleeve condition that represent the anthropometric spectrum for integrating into CAD designs of the CEV seat would allow for a seat pan design that accommodates the total variation in the human form.

\subsection{Methods}

An anthropometric survey of 33 (16 males, 17 females) participants was conducted to capture the specific anthropometric measurements critical to the seat-pan including hip breadth, buttock-popliteal length, and popliteal length. Using the anthropometric data, a subset of these participants $(n=7)$ was chosen as representative of the $1^{\text {st }}, 50^{\text {th }}$, and $99^{\text {th }}$ percentile critical anthropometric values (Table 5) to capture the contour of the lower-body. These percentile values were based on a modified age truncated ANSUR database population $[5,6]$. Due to time constraints, not all $1^{\text {st }}, 50^{\text {th }}$, and $99^{\text {th }}$ data values for each measurement could be exactly matched, but individuals were selected that had the closest value to the percentile data (Table 6).

Table 5. Male and female data representing the $1^{\text {st }}, 50^{\text {th }}$, and $99^{\text {th }}$ percentiles of the population.

\begin{tabular}{|l|c|c|c|c|c|c|}
\hline \multirow{2}{*}{ Percentile } & \multicolumn{2}{|c|}{$\begin{array}{c}\text { Hip Breadth, Sitting } \\
\text { [cm (in)] }\end{array}$} & \multicolumn{2}{c|}{$\begin{array}{c}\text { Buttock-Popliteal Length } \\
\text { [cm (in)] }\end{array}$} & \multicolumn{2}{c|}{$\begin{array}{c}\text { Popliteal Length } \\
\text { [cm (in)] }\end{array}$} \\
\cline { 2 - 7 } & Female & Male & Female & Male & Female & Male \\
\hline $1^{\text {st }}$ & $32.7(12.9)$ & $31.5(12.4)$ & $42.2(16.6)$ & $44.6(17.6)$ & $33.2(13.1)$ & $38.3(15.1)$ \\
\hline $50^{\text {th }}$ & $39.6(15.6)$ & $37.5(14.8)$ & $48.5(19.1)$ & $51.0(20.1)$ & $38.6(15.2)$ & $44.1(17.4)$ \\
\hline $99^{\text {th }}$ & $46.5(18.3)$ & $43.4(17.1)$ & $54.8(21.6)$ & $57.3(22.6)$ & $44.0(17.3)$ & $50.0(19.7)$ \\
\hline
\end{tabular}

Table 6. Seat Pan Participant Critical Anthropometric Data $(n=7)$.

\begin{tabular}{|c|c|c|c|c|}
\hline $\begin{array}{c}\text { Subject } \\
\text { ID }\end{array}$ & Gender & $\begin{array}{c}\text { Hip Breadth, Sitting } \\
{[\mathrm{cm} \text { (in)] }}\end{array}$ & $\begin{array}{c}\text { Buttock-Popliteal Length } \\
{[\mathrm{cm}(\mathrm{in})]}\end{array}$ & $\begin{array}{c}\text { Popliteal Length } \\
{[\mathrm{cm} \text { (in)] }}\end{array}$ \\
\hline 18 & $\mathrm{~F}$ & $45.5(17.9)^{\star \star \star}$ & $55.0(21.7)$ & $44.8(17.6)$ \\
\hline 19 & $\mathrm{~F}$ & $40.3(15.9)^{\star *}$ & $53.9(21.2)$ & $43.3(17.0)$ \\
\hline 25 & $\mathrm{~F}$ & $31.5(12.4)^{\star}$ & $49.5(19.5)$ & $42.1(16.6)$ \\
\hline 30 & $\mathrm{~F}$ & $33.8(13.3)$ & $45.2(17.8)^{\star}$ & $38.5(15.2)^{\star}$ \\
\hline 5 & $\mathrm{~F}$ & $40.7(16.0)$ & $50.8(20.0)^{\star *}$ & $39.9(15.7)$ \\
\hline 21 & $\mathrm{M}$ & $38.0(15.0)$ & $50.0(19.7)$ & $44.4(17.5)^{\star \star}$ \\
\hline 32 & $\mathrm{M}$ & $37.8(14.9)$ & $57.1(22.5)^{\star \star \star}$ & $49.7(19.6)^{\star \star \star}$ \\
\hline
\end{tabular}

*Selected participant anthropometric value closest to the $1^{\text {st }}$ percentile

** Selected participant anthropometric value closest to the $50^{\text {th }}$ percentile

${ }^{* * *}$ Selected participant anthropometric value closest to the $99^{\text {th }}$ percentile

The seven subset participants were scanned using a 3D full-body laser scanner. Participants were scanned in a seat mock-up, configured in an expected flight-like position inside the vehicle cockpit with similar hip joint and knee joint angles. The seat pan was removed to allow the laser scanner to capture the contour of the participant's lower body. Each 3D surface scan was then imported into a CAD software package for further analysis.

\subsection{Results}

Seven subjects were scanned in the seat mockup (Figure 5). The surface scans were processed by removing the extraneous seat mockup hardware, and adding reference plans for the seat pan and back rest to aid in alignment of the scans and placement within the CAD vehicle/seat environment. Figure 5 (right) contains an example of the finished and referenced participant's surface scan image. The seven surface scans were overlaid using the reference planes in CAD to compare and contrast the varying curvatures of the lower body (Figure 6). Capturing the curvature of the lower-body representative of potential crewmember anthropometry serves as a design tool and was provided through the 3D surface scans. The overlaid surface scans were then used in the fabrication of a contoured seat-pan mockup chair. 

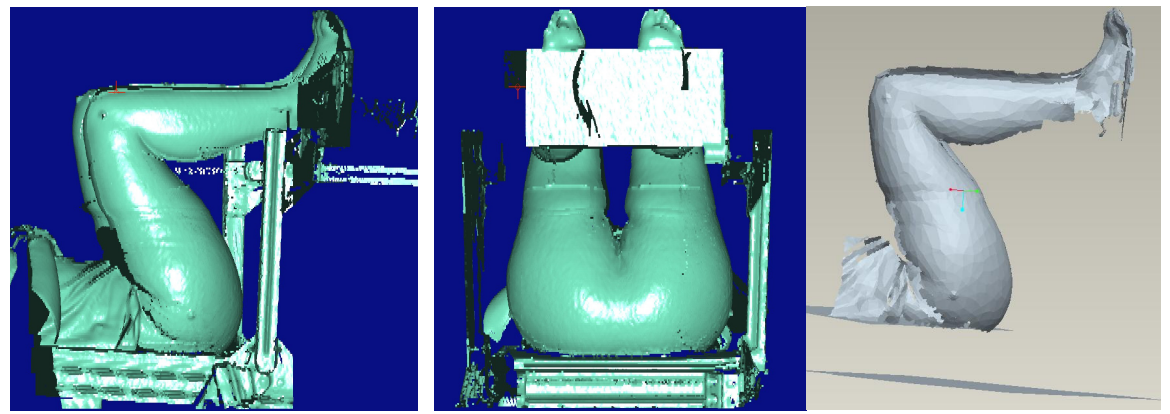

Fig. 5. Participant surface scan raw (left, middle) and surface mesh in CAD (right).
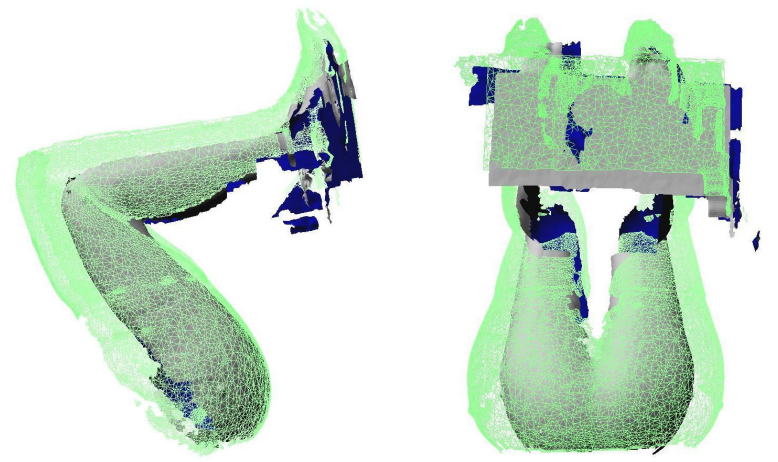

Fig. 6. Two overlaid, aligned body surface scans.

\subsection{Summary}

Measurements such as hip breadth, buttock-popliteal height, and popliteal length cannot be used to reconstruct and represent the curvature of the lower body. Even with the volumetric information on hand, it is still difficult to quantify a surface mesh as representative of the entire user population. The linear measurements from an individual can be mapped to their 3D scans to place the scans within the context of the population for evaluation of the seat contour.

\section{Case Study \#4: Boundary Manikin Generation}

To validate if a suit satisfies sizing requirements and accommodates the range of the population, it must be thoroughly tested with many different types of body morphology. However, this can be a costly trial and error process if performed after the suits have been manufactured. An alternative is to provide 3D scans that represent the ranges of body types the suit designer will have to accommodate. Unfortunately, there is limited surface scan data that matches the entire range of measurements required for the database of interest [5]. Thus, the idea is to modify existing surface scans (baseline scans) using the relationship of baseline anthropometry to targeted anthropometry to generate 3D manikins for use with CAD. This section focuses on the ability to 'morph' a 3D laser scan of an individual to a whole new set of anthropometry and the resulting development of $3 \mathrm{D}$ digital manikins.

\subsection{Background}

Boundary manikins, in the context of this paper, are representative digital replicas of real subjects' anthropometry from a database that spans the $1^{\text {st }}$ to $99^{\text {th }}$ percentile measurements critical to suit fit relative to the database of interest [5]. The details on the selection of anthropometry and derivation of the boundary manikin anthropometry have been previously published $[9,10]$. This boundary manikin anthropometry is used to transform a baseline subject scan into a representative 3D digital manikin with the corresponding anthropometric values. These 3D manikins can then serve as templates that can aid in the design of the future spacesuits.

\subsection{Methods}

\subsubsection{Baseline scans and anthropometry}

The ability to morph an individual from one set of anthropometry to another requires a certain subset of anthropometry to fully adjust a scan. The measurements used (Table 7) were based on available measurements collected through the standard anthropometric data collection performed at the ABF [2] 
and the measurement selection itself also corresponded to the available body dimensions in the modified age truncated ANSUR database, representing the astronaut population $[5,6]$. These measurements were selected to represent the length, width, and breadths of discrete body landmarks readily identifiable from the scanner images; if widths/breadths were not readily available the circumferential measurement was used instead.

Table 7. Measurements for morphing the human body.

\begin{tabular}{|l|l|}
\hline Acromion-Radiale Length & Hip Breadth \\
\hline Biacromial Breadth & Lateral Femoral Epicondyle Height \\
\hline Biceps Circumference, flexed & Lateral Malleolus Height \\
\hline Calf Circumference & Radiale Stylion Length \\
\hline Cervicale Height & Thigh Circumference \\
\hline Chest Breadth & Trochanterion height \\
\hline Chest Depth & Waist Breadth \\
\hline Forearm Circumference, flexed & Waist Depth \\
\hline Gluteal Furrow Height & \multicolumn{1}{|l}{} \\
\cline { 2 - 2 }
\end{tabular}

To collect this baseline anthropometry and the associated scans to perform the morphing procedure, markers were placed on specific landmarks of the scanned subjects consistent to the required measurements and two scans were collected in various postures using a VITUS 3D Laser Scanner. Two scanner operators extracted the anthropometry listed in Table 7 and the results were averaged; the multiple extractors were used to minimize human error in the measurements. To morph the baseline scan to a new manikin, a reference T-pose scan was gathered in addition to the anthropometric information collected by the scanner. The T-pose scan of each subject was exported to Polyworks for hole filling, segmentation, and post processing for importation into a custom MATLAB (The MathWorks Inc. Natick, MA) program.

\subsubsection{Scanned subjects}

Originally, the proposed analysis called for matching up the anthropometry of the boundary manikins as close as possible to the baseline scans. However, as the final results from the PCA and Whole Body Posture Based Analysis (WBPBA) [9,10] were realized, it became unrealistic to find the variety of subjects close to all dimensions needed for the morphing procedure. As the boundary manikins' anthropometry spans the entire range of the population across multiple measurements, it is not pragmatic to conduct an anthropometric survey of a multitude of subjects to gather their anthropometry in the hopes of striking close to the target anthropometry and body shape of all the proposed manikins. Instead, a key element involving the body somatotypes of the torso was examined, namely the volumetric consideration of the shape and distribution of weight. It was hypothesized that the human torso would be the trickiest portion of the body to deform, since the distribution of weight varies widely from person to person, and if the scans could be matched to body somatotypes, the deformations of the torso region would be more realistic.

Due to the skin deformation present in the torso as weight varies, it was desired that a range of baseline scans covering these somatotypes were to be collected. These somatotypes were identified by looking at the ratios of the chest breadth, waist breadth, and hip breadth. Somatotypes of spoon, pear, hourglass, and ruler were identified in the boundary manikin anthropometry. A spoon has an equal chest breadth and waist breadth ratio, with a larger hip breadth. A ruler has equal ratios of all three dimensions. A pear has increasing ratios from the chest down through the waist to the hip breadths, and the hourglass has a small waist breadth in comparison to the hip and chest breadths. In the boundary manikin anthropometry, the females had hourglass, spoon, and pear somatotypes and the males had hourglass, ruler, spoon, and pear somatotypes.

A total of 6 baseline subjects were used in the analysis, three males and three females, to cover the range of body somatotypes. Baseline subjects were identified and scanned in a reference T-pose scan, who matched the representative somatotypes and they were then paired with the corresponding boundary manikins. Due to a lack of available pear somatotypes in the male baseline subject selection, the pear boundary manikin anthropometries were coupled with the spoon anthropometry due to the similarities in the magnitude of their anthropometry. 


\subsubsection{Manikin anthropometry}

The results from the PCA yielded 20 female manikins and 22 male manikins. The WBPBA resulted in a total of four female and three male manikins $[9,10]$. Therefore, there were 24 female and 25 male boundary manikin anthropometries generated from the two analyses that could be generated in the morphing procedure.

While the initial number of 49 manikins provided a comprehensive representation of the various anthropometry combinations, there was a concern that all the manikins ultimately might not be used by the designer because of the vast amount. Therefore, the number of manikins was reduced down to a viable representative subset of 11 out of the 49 manikins via an optimization method to identify key manikins for the most critical anthropometric measurements. This optimization method reduced the total number of manikins while ensuring the maintenance of a suitable representation of the target population range. The lesser amount of manikins will also serve to promote the use of the boundary manikins by the designer to assist in the suit design process while not overwhelming them. The results of the truncation produced six female and five male boundary manikin anthropometries to generate using the morphing tool.

It is critical to note that the head, hands, and feet of the manikins were not adjusted volumetrically. The scope of the project required the manikins to assist in the validation of whether a suit satisfies the sizing requirements and did not pertain to fitting the helmet, gloves, or boots due to the very specific measurements and postures required to size these components. In addition, the morphing of the head would have resulted in an erroneous result due to the complex geometric structure of the head-neck complex and the inability to segment the head into various pieces to account for all the minute details. Similarly, the foot is a complex shape that requires far more measurements to accurately deform. The hands were scanned with the fingertips and thumb grouped together and a change in the hand length or circumference would have been universally applied to the entire hand, resulting in an inaccurate morph. As a result, the baseline subjects head, hands, and feet were not morphed on the resulting manikin. They are included as a reference to assist in the placement of bearings or other suit components at the wrist, neck, and ankles; however, they are not intended to be used for design purposes. The minor variations between the hands and feet in the final boundary manikins are due to variances in the resolution of the scan when regenerating the 3D mesh for export into a CAD model.

\subsubsection{Morphing methodology}

Morphing a scan involves the application of manikin geometry to a body scan; the body scan is adjusted volumetrically to represent the manikin geometry. After conducting an extensive literature review it was decided that the morphing would be accomplished via the deformation of scanner point cloud information. A 3D scan is composed of a surface mesh of triangles. The vertices of these triangles were saved and imported into MATLAB as a point cloud of data in $3 D$ space. These points were divided into various body segments and tagged with the landmarks of the measurements of interest.

The morphing procedure involved a MATLAB program custom written by the ABF that manipulated the point cloud of a baseline subject. Essentially, a reference point was selected, either a landmark (for length) or the centerline through a segment (for breadths, lengths, and circumferences). The vector formed between that reference point and a point within the cloud had a scaling factor applied along the relevant axial system (Eqn. 1). The scaling factors (Eqn. 2) are the ratios of baseline scan anthropometry to target boundary manikin anthropometry. After the length morphing, the positions of all the 'child' segments relative to the morphed parent were altered to compensate for the morphing adjustment. For example, if the upper leg was lengthened the lower leg and foot was shifted so the transition between the two segments remained congruent.

$$
\begin{aligned}
& \text { NewPtCloud }_{i x}=\text { RfPt }_{x}+\left(\text { PtCloud }_{i x}-\text { RfPt }_{x}\right) * \text { ScalingFactor } \\
& \text { ScalingFactor }=\frac{T \arg \text { etAnthro }}{\text { BaselineAnthro }_{x}}
\end{aligned}
$$

The length morphing used Equation 1 with $x$ as the long axis of the segment and $i$ the relevant point in the point cloud (Figure 7). The crotch landmark was the total body reference point, to align all the morphed scans and to provide an overall reference point for the shifting of body segments. 

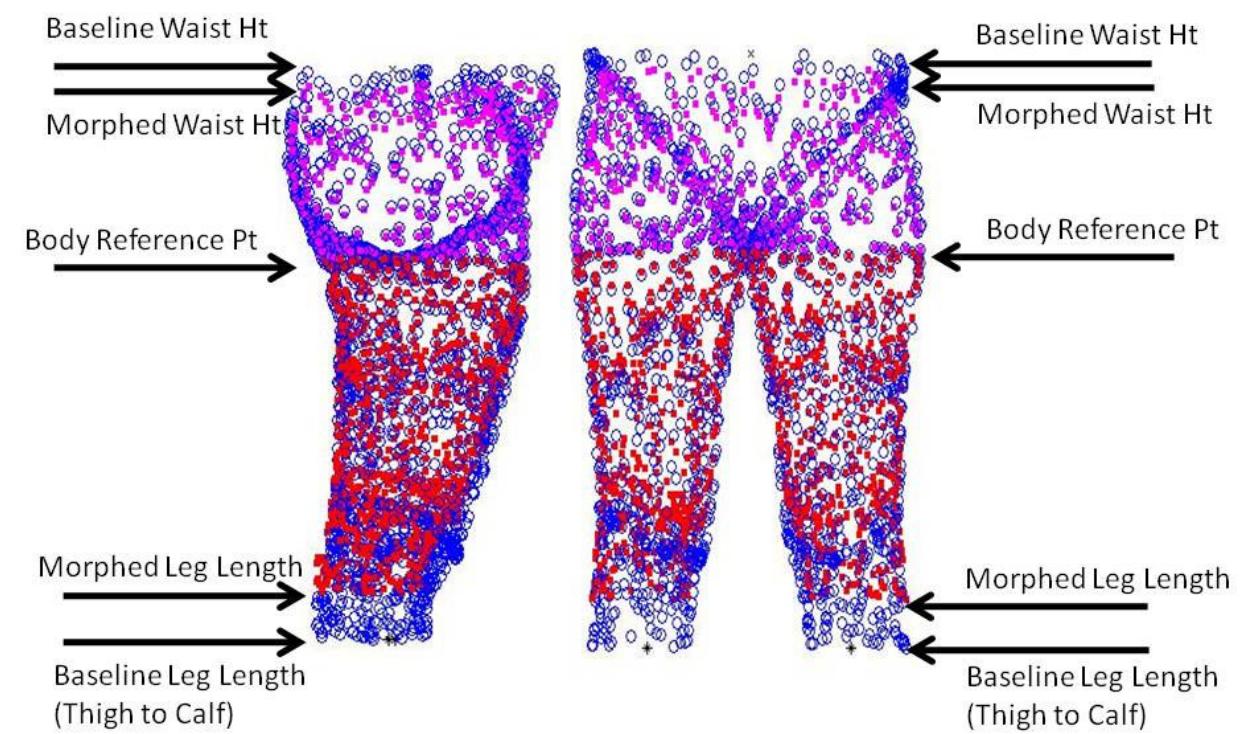

Fig. 7. Baseline (blue circles) and morphed image (red, purple squares) illustrating length morphing.

For the body width and breadth anthropometry, Equation 1 was used with $x$ as either the anterior-posterior or mediolateral axes of the segment and $i$ the relevant point in the data cloud (Figure 8). For example, to apply a change in chest breadth to a baseline subject, the chest is widened at the level of the bust point by taking the point on the chest segment and multiplying the scaling factor (Eqn 2) on the magnitude of the vector formed by the point with respect to the center of the chest. This results in the points closest to the origin to be adjusted minimally in relation to the points farther away as a function of distance from the origin for a given segment.

After this initial methodology for the breadths and widths was attempted and validated, a problem became apparent. If, for example, the chest and waist breadths were both adjusted with unique scaling factors, it would result in disconnects between the point clouds of the two segments (Figure 8). This problem was solved by creating a scaling factor that changed as a function of distance away from the landmark origin along the length of the segment. With the previous chest breadth example, instead of applying a continuous scaling factor for all points in the chest segment until the waist segment was reached, the scaling factor would taper exponentially to a value of 1 as it moved away from the bust point down the torso until it reached the boundary between the chest and waist segments. However this caused problems as well because the boundary between the two segments therefore never had a scaling factor applied, resulting in large portions of the body unchanged. Ultimately, the body was re-segmented using the known width- and breadth-related landmarks as the segment boundaries. The scaling factor was then exponentially tapered to a value of 1 as it reached the next anthropometric measurement using Equation 3, where $x$ is the anterior posterior or mediolateral axis, $z$ as the long axis of the segment, and $i$ the relevant point in the data cloud. In the case of the chest breadth example, the chest breadth scaling factor would taper exponentially to a value of 1 going from the bust point to the navel. When the waist breadth was then adjusted, the waist breadth scaling factor would conversely impact the torso by applying the exponentially tapering scaling factor up from the navel to the bust point. In this manner, all measurement changes resulted in a smooth transition between segments and the ability to individually adjust single measurements without impacting other critical anthropometric measurements.

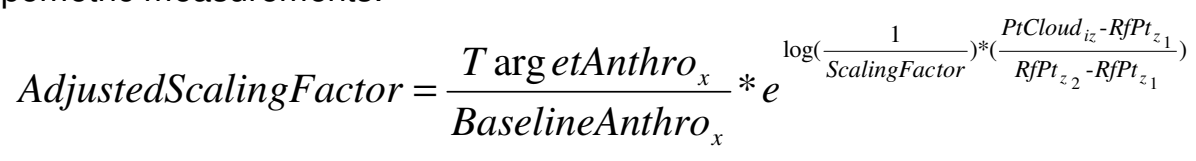



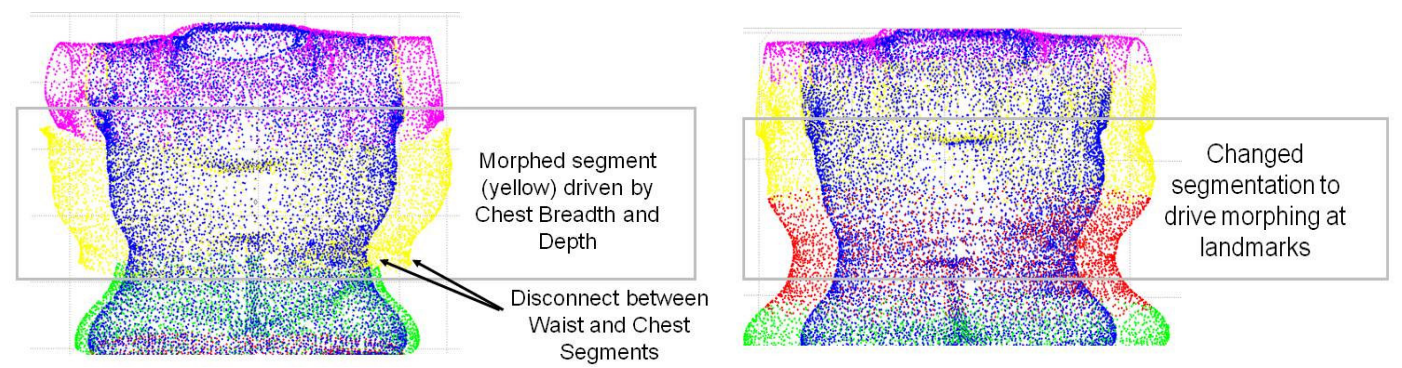

Fig. 8. Baseline (blue) and morphed image (red, yellow, purple, green) illustrating smoothing between segments.

The width, breadth, and length manipulations of each segment were combined into an overall morphed image (Figure 9). This new point cloud data was then exported into Polyworks and a 3D surface mesh was regenerated for export into other CAD model programs.

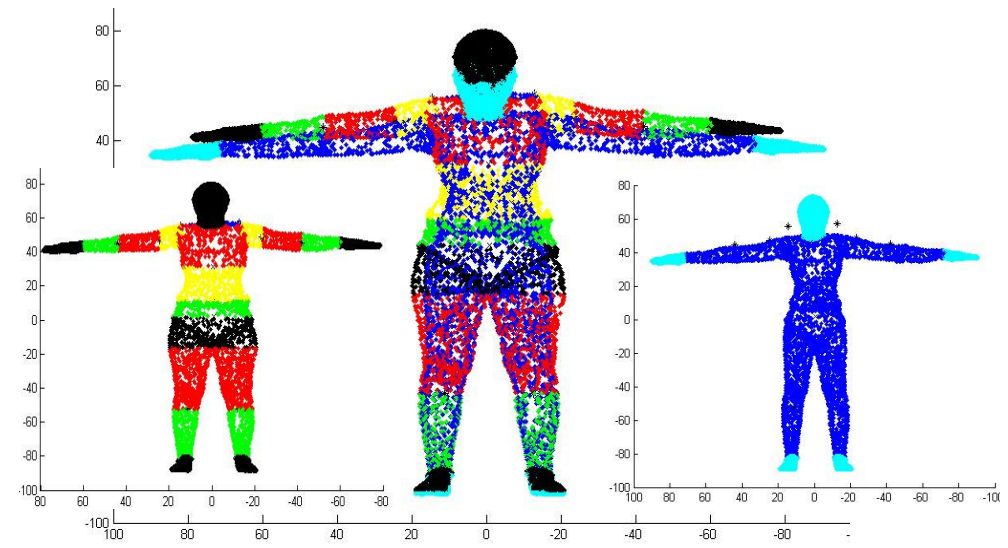

Fig. 9. Baseline (left - red, yellow, green, black) and final point cloud of morphed image (right-blue).

\subsection{Results}

The morphing tool has capabilities to provide a volumetric representation of real subject anthropometry without necessarily scanning a subject who has that anthropometry. The 11 manikins generated by this analysis are shown in Figure 10. To ensure the accuracy of the morph, the manikin anthropometry was verified by extracting the anthropometry from the scan and comparing those measurements to the target anthropometry. Thus, the final result is a verified representative manikin for use with sizing, along with the linear anthropometric measurements that are tied to each manikin for use in analytical analyses. The results from this process can be used in design process modeling and initial suit sizing work as 3D, realistic examples of individuals from the population, while maintaining the variability between and correlation with the dimensions of interest.

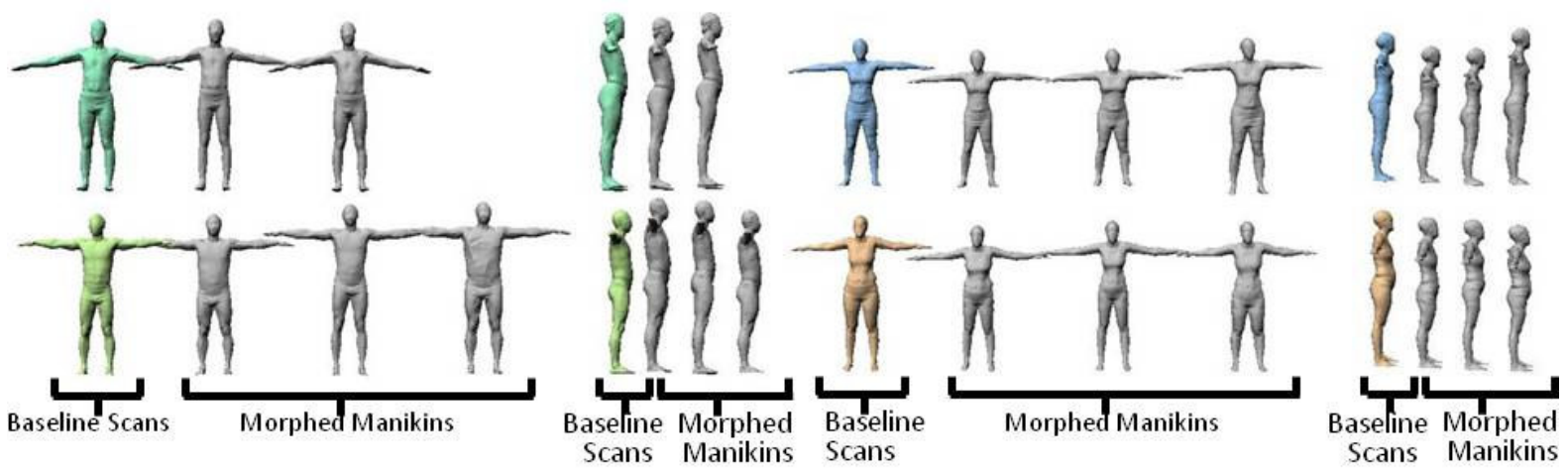

Fig. 10. The final 11 morphed manikins and each baseline scan. 


\section{Conclusion}

The adoption of three dimensional laser scanning has led to an increase in the capabilities of human factors analyses related to space vehicle and spacesuit design. The ability to provide information on volume and shape of the human body can augment traditional human factors analysis with better tools set for predicting and analyzing the human within the spacesuit and vehicle. In the case studies provided, scans of the human body coupled with linear based dimensions have led to increased understanding of multivariate characteristics of the head, suit-human interface, and the conformal nature of the human body. The culmination of this linear to volumetric based relationship resulted in the ability to morph baseline scans into new scans representing a set of target anthropometry. These methodologies will assist in the improvement of human-system interactions, the evaluation of accommodation parameters, and the further development of new processes to aid in the design and evaluation of space systems.

\section{References}

1. McConville, J., Churchill, T., Kaleps, I., Clauser, C., Cuzzi, J. (1980): "Anthropometric Relationships of Body and Body Segment Moments of Inertia", Air Force Aerospace Medical Research Laboratory, Aerospace Medical Division, Air Force Systems Command, AFAMRL-TR-80-119, Wright-Patterson Air Force Base, Ohio.

2. Ferrer, M. A., Young, K. S., Margerum, S. (2006): "Suit Specific Anthropometric Data Extraction Procedures Manual", JSC 63439 Rev A (2006), NASA, Johnson Space Center, Houston, Texas.

3. Robinette, K.M., Blackwell, S., Daanen, H.A.M., Fleming, S., Boehmer, M., Brill, T., Hoeferlin, D., Burnsides, D., (2002): "Civilian American and European surface anthropometry resource (CAESAR)", Final Report, Summary, Vol. I. AFRL-HE-WP-TR-2002-0169.

4. Blackwell, S., Robinette, K.M., Daanen, H.A.M., Boehmer, M., Fleming, S., Kelly, S., Brill, T., Hoeferlin, D., Burnsides, D., (2002): "Civilian American and European surface anthropometry resource (CAESAR)", Final Report, Descriptions, Vol. II, AFRL-HE-WP-TR-2002-0170.

5. CxP 70024. Rev. D. (2009): "NASA Human Systems Integration Requirements (HSIR)", Houston, TX.

6. Gordon, C.C., Bradtmiller, B., Churchill, T., Clauser, C.E., McConville, J.T., Tebbetts. I., Walker, R.A. (1989): "1988 Anthropometric Survey of U.S. Army Personnel: Methods and Summary Statistics", U.S. Army NATICK RD\&E Center. NATICK/TR-89/044.

7. Zehner, G. (2000): "Prediction of Anthropometric Accommodation in Aircraft Cockpits", Ohio State University. [dissertation]

8. Loker, S., Ashdown, S. P., Schoenfelder, K. (2005): "Size-specific analysis of body scan data to improve apparel fit", Journal of Textile and Apparel, Technology and Management, 4(3).

9. Young, K., Margerum, S., Barr, A., Ferrer, M., Rajulu, S. (2008): "Generation of Boundary Manikins Anthropometry", International Conference on Environmental Systems.

10. Young, K., Margerum, S., Barr, A., Ferrer, M., Rajulu, S. (2008): "Derivation of Boundary Manikins: A Principal Component Analysis", Digital Human Modeling for Design and Engineering Conference. 